Check for updates

Cite this: Phys. Chem. Chem. Phys., 2020, 22, 10641

Received 14th November 2019, Accepted 9th December 2019

DOI: $10.1039 / c 9 c p 06161 d$

rsc.li/pccp

\section{Quantifying the hydration structure of sodium and potassium ions: taking additional steps on Jacob's Ladder}

\author{
Timothy T. Duignan, (D)*ab Gregory K. Schenter, (D) a John L. Fulton, ${ }^{a}$ \\ Thomas Huthwelker, ${ }^{c}$ Mahalingam Balasubramanian, ${ }^{d}$ Mirza Galib, (D) ${ }^{a}$ \\ Marcel D. Baer, (D) ${ }^{a}$ Jan Wilhelm, ${ }^{\text {ef }}$ Jürg Hutter, $^{e}$ Mauro Del Ben, ${ }^{9}$ X. S. Zhao (iD $* b$ \\ and Christopher J. Mundy (D)*ah
}

\begin{abstract}
The ability to reproduce the experimental structure of water around the sodium and potassium ions is a key test of the quality of interaction potentials due to the central importance of these ions in a wide range of important phenomena. Here, we simulate the $\mathrm{Na}^{+}$and $\mathrm{K}^{+}$ions in bulk water using three density functional theory functionals: (1) the generalized gradient approximation (GGA) based dispersion corrected revised Perdew, Burke, and Ernzerhof functional (revPBE-D3) (2) the recently developed strongly constrained and appropriately normed (SCAN) functional (3) the random phase approximation (RPA) functional for potassium. We compare with experimental $X$-ray diffraction (XRD) and X-ray absorption fine structure (EXAFS) measurements to demonstrate that SCAN accurately reproduces key structural details of the hydration structure around the sodium and potassium cations, whereas revPBE-D3 fails to do so. However, we show that SCAN provides a worse description of pure water in comparison with revPBE-D3. RPA also shows an improvement for $\mathrm{K}^{+}$, but slow convergence prevents rigorous comparison. Finally, we analyse cluster energetics to show SCAN and RPA have smaller fluctuations of the mean error of ion-water cluster binding energies compared with revPBE-D3.
\end{abstract}

\section{Introduction}

The sodium and potassium ions play a central role in a large range of important industrial and biological processes. For example, the sodium and potassium ions are considered to be promising candidates to replace lithium ions in the next generation of energy storage devices. ${ }^{1-5}$ Additionally, the flow of potassium and sodium ions through cell membranes is used to control important biological processes. ${ }^{6}$ In both of these systems, an important step is the partial desolvation of the ion

\footnotetext{
${ }^{a}$ Physical Science Division, Pacific Northwest National Laboratory, P. O. Box 999, Richland, Washington 99352, USA. E-mail: chris.mundy@pnnl.gov

${ }^{b}$ School of Chemical Engineering, The University of Queensland, St Lucia, Brisbane 4072, Australia.E-mail: t.duignan@uq.edu.au, george.zhao@uq.edu.au ${ }^{c}$ Swiss Light Source, Paul Scherrer Institut (PSI), 5232 Villigen, Switzerland

${ }^{d} \mathrm{X}$-ray Sciences Division, Advanced Photon Source, Argonne National Laboratory, Lemont, Illinois 60439, USA

${ }^{e}$ Department of Chemistry, University of Zurich, CH-8057 Zürich, Switzerland

${ }^{f}$ Institute of Theoretical Physics, University of Regensburg, D-93053 Regensburg, Germany

${ }^{g}$ Computational Research Division, Lawrence Berkeley National Laboratory, Berkeley, California 94720, USA

${ }^{h}$ Department of Chemical Engineering, University of Washington, Seattle, Washington, USA
}

as it passes through a small channel. For example, the ions must desolvate to intercalate into electrode materials or to pass through the ion pump in the cell membrane. This desolvation can determine the rates and mechanisms of these processes. A recent demonstration of this point is provided by Peng et al. ${ }^{7}$ who demonstrate that interfacial sodium ion diffusion is highly sensitive to the hydration number. It is therefore very important to build an accurate and detailed understanding of the structure of solvent around these ions.

Many attempts have been made to simulate the properties of these ions in water. ${ }^{8-21}$ Classical forcefield molecular dynamics can reproduce a variety structural properties such as the peak position in the radial distribution function (RDF) but the parameters for the Lennard-Jones and polarisability interactions have to be explicitly adjusted to do so. These models have limited predictive power as new parameters are usually needed to model the ion in different environments such as inside an electrode material. A promising approach is to build force fields based on exhaustive fitting to reproduce cluster energies. ${ }^{22-24}$ However, this approach requires extensive human and computational time to develop the new potentials.

Hybrid quantum mechanical/molecular mechanics (QM/MM) is an alternative approach ${ }^{14,21}$ to the problem. However, it is 
unclear how large the QM region needs to be to accurately capture the full solvent structure. Moreover, significant challenges arise associated with the treatment of the interface between the classical and quantum regions.

A promising and increasingly widely used approach is to use molecular dynamics simulations with a full quantum mechanical density functional theory treatment of the system. ${ }^{25}$ The dispersion corrected generalized gradient approximation (GGA) functionals are by far the most widely used in the context of condensed phase simulation due to their low computational demand. Of these functionals the revised Perdew, Burke, and Ernzerhof functional with Grimme dispersion correction (revPBE-D3) ${ }^{26-28}$ has been demonstrated to reasonably accurately reproduce the structure of bulk water. ${ }^{29}$ However, the predictions of this approach often depend sensitively on the specific functional chosen and can therefore fail to reproduce experimental measurements quantitatively. For example, Galib et $a .^{20,30}$ demonstrated that standard GGA functionals cannot reproduce the experimentally determined water structure around the sodium cation as determined by X-ray diffraction (XRD) and X-ray absorption fine structure measurements (EXAFS). In the case of monovalent ions in solution, the details of the aqueous response to an ion can be shown to be a necessary component to predicting the single-ion free energies. ${ }^{31-34}$

Obviously, all density functional theory (DFT) functionals involve significant approximations and therefore no one functional can be expected to work perfectly for all applications. However, it is important to identify which functionals perform best for a given property of interest. Where the accuracy is determined to be sufficient we can gain useful physicochemical insight. The focus of this study is to move toward an understanding of what representations of interaction are necessary to obtain quantitative agreement with measurements probing local solvation structure. We will demonstrate that monovalent cations can be simulated at significantly higher accuracy using higher rungs of the so-called "Jacobs Ladder" of DFT functionals. ${ }^{35}$

Interestingly, for the cations considered in this study, no significant change in agreement between simulation and experiment occurs when moving from the GGA functional PBE to the hybrid functional PBE0, ${ }^{17}$ indicating that hybrid functionals are unlikely to contain the correct physics to describe ion solvation. The strongly constrained and appropriately normed (SCAN) meta-GGA functional has recently been developed in hope of obtaining chemical accuracy for complex condensed phase systems. Taking this single step up the Jacobs Ladder has the advantage that the computational costs are not too much higher than for standard GGA functionals. SCAN has been developed to satisfy 17 known constraints that a general exchange-correlation functional should satisfy. It can accurately reproduce binding energies and structures of a variety of molecules without empirical dispersion corrections. ${ }^{36,37}$ This functional has also recently been applied to calculate the potential of mean force of the $\mathrm{NaCl}$ dimer in water. ${ }^{38}$

Additionally, the random phase approximation (RPA) to electron correlation has recently been implemented in $\mathrm{CP}_{2} \mathrm{~K}^{39}$ for condensed phase calculations. ${ }^{40,41}$ This level of theory represents the highest (fifth) rung of the Jacobs Ladder and provides an accurate description of bulk liquid water. ${ }^{41}$ The RPA method comes with additional computational costs over standard functionals due to the explicit treatment of electron correlation and the requirement of larger basis sets. This study will provide an important step forward towards quantitatively determining whether more sophisticated treatments of electron correlation can overcome the limitations of the GGA based description of ion solvation.

\section{Structural analysis}

Pure water structure. One difference between this study and the studies of Chen et $a l .{ }^{42}$ and Zheng et $a l .{ }^{43}$ is the present work performs simulations at $300 \mathrm{~K}$ rather than $330 \mathrm{~K}$. In addition, newly optimized pseudo potentials for the oxygen atom are used. We use the standard PBE pseudo potentials for hydrogen. ${ }^{44}$ Chen et $a .^{42}$ also use the isothermal-isobaric ensemble (constant NpT) in contrast with the canonical (NVT) ensemble used here and by Zheng et al. ${ }^{43}$

The resulting oxygen-oxygen RDFs are shown in Fig. 1 for both revPBE-D3 and SCAN and compared with experimental XRD data. ${ }^{45}$ As has been reported before it is clear that the revPBE-D3 RDF is closer to experiment. ${ }^{29,46}$ This is partially the result of a small cancellation of errors associated with the neglect of nuclear quantum effects and errors in the revPBE-D3 functional. ${ }^{46}$ The structure is significantly more enhanced for the SCAN functional compared with experiment. It resembles ${ }^{47}$ the structure produced with the GGA functional of Becke ${ }^{48}$ and Lee, Yang and Parr $^{49}$ (BLYP) with the Grimme dispersion correction (D2). ${ }^{50}$ The origin of the discrepancy in comparison with previous SCAN studies ${ }^{42,43}$ is likely due to the $30 \mathrm{~K}$ higher temperature used in these studies. This higher temperature was used to account for the effect of nuclear quantum effects. The effects associated with quantum nuclear effects on the oxygenoxygen RDF for water have been shown to be quite small for high quality interaction potentials. ${ }^{51-53}$ The slightly over-structured behaviour of SCAN observed here is consistent with the work of Wiktor et al. ${ }^{54}$ and Yao and Kanai ${ }^{38,55}$ who also simulated at $300 \mathrm{~K}$. Yao and $\mathrm{Kanai}^{38,55}$ also used both $\mathrm{CP}^{2} \mathrm{~K}^{56}$ and $\mathrm{CPMD}^{57}$ demonstrating good agreement between the two basis set approaches, namely Gaussian functions and plane-waves. Simulation of $20 \mathrm{ps}$ utilizing SCAN water in a slab configuration at $300 \mathrm{~K}$ using the

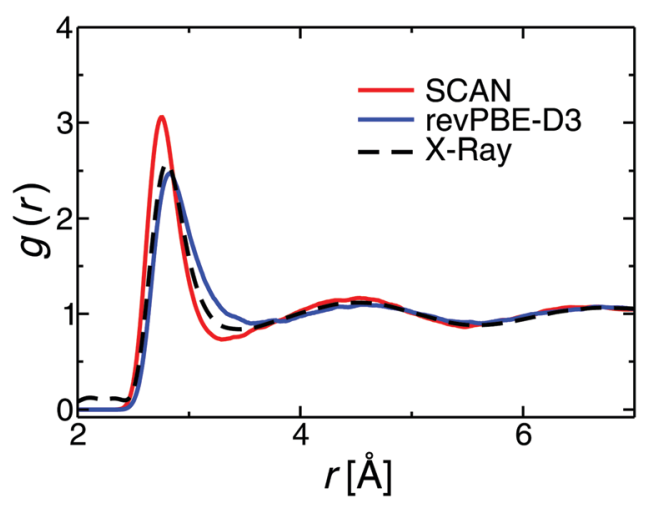

Fig. 1 Oxygen-oxygen RDFs with the revPBE-D3 and SCAN functionals compared with experimental XRD data. ${ }^{45}$ 


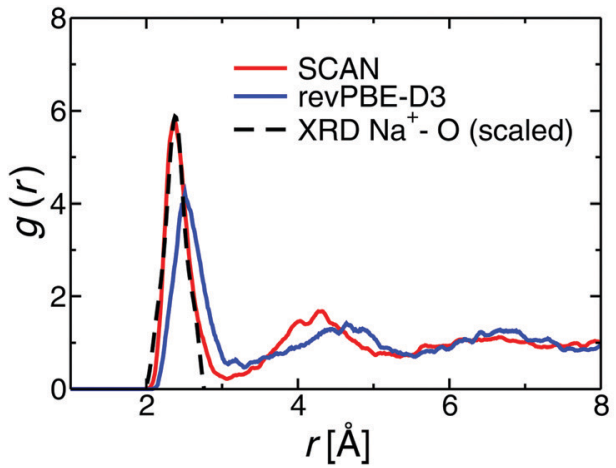

(a) Sodium $\left(\mathrm{Na}^{+}\right)$

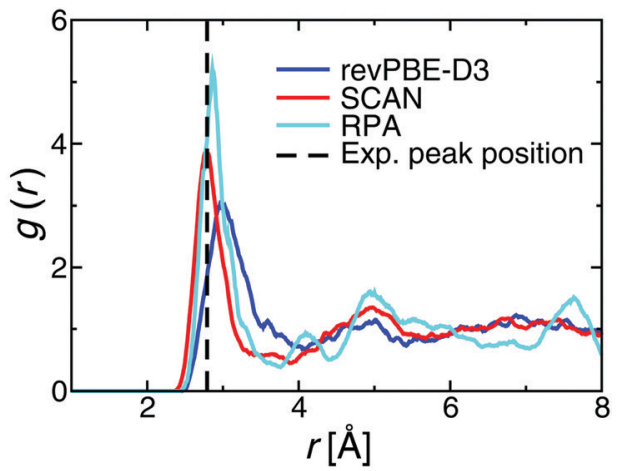

(b) Potassium $\left(\mathrm{K}^{+}\right)$

Fig. 2 RDFs of solvated sodium and potassium ions in water with the revPBE-D3 and SCAN functionals demonstrating that the SCAN functional reproduces the experimentally observed peak position $\left(\mathrm{Na}^{+}, \mathrm{K}^{+}\right)$and peak shape $\left(\mathrm{Na}^{+}\right)$much more accurately.

protocol discussed herein is consistent with the density of $1.05 \mathrm{~g} \mathrm{~cm}^{-3}$ calculated by Chen $e t a l^{42}$ and Wiktor et al. ${ }^{54}$ This density is larger than the experimental density $\left(0.997 \mathrm{~g} \mathrm{~cm}^{-3}\right)$. These results suggest some caution is appropriate in the use of SCAN to describe pure water.

Cation hydration structure. Fig. 2 depicts the RDF for the sodium and potassium ions utilizing both revPBE-D3 and SCAN functionals. Significant differences are apparent. For $\mathrm{Na}^{+}$we compared with the rescaled $\mathrm{Na}^{+}-\mathrm{O}$ peak extracted from XRD of $\mathrm{NaCl}$ at $6 \mathrm{M}^{20}$ and for $\mathrm{K}^{+}$we compare with the experimentally determined peak position. ${ }^{58-60}$

As shown in Table 1, the experimental cation-oxygen peak position is reproduced much more accurately with the SCAN functional than with the GGA functional. The inability to reproduce this peak position has been problematic with other GGA functionals such as BLYP-D2. ${ }^{20}$ To the best of our knowledge, this is the first demonstration that the SCAN functional significantly outperforms standard GGA functionals in reproducing bulk structural properties of ions in the condensed phase. As previously stated, earlier research has argued that SCAN can accurately reproduce bulk water structure ${ }^{42,43}$ but this appears to require unphysically elevating the temperature by $+30 \mathrm{~K}$. Similarly, the $\mathrm{Na}-\mathrm{Cl}$ potential of mean force (PMF) in water has been computed with SCAN. ${ }^{38}$ SCAN doesn't appear to reproduce the NaCl dimer binding energy in vacuum as accurately as the wB97X-V functional. ${ }^{38}$ However, relatively small differences are observed when SCAN is compared to other traditional DFT functionals and there are no direct comparisons of the outcomes of the PMF with experimental results to demonstrate any improvement. Although, we

Table 1 RDF peak positions for different DFT functionals compared with experiment demonstrating that the SCAN functional provides closer agreement to that measured by X-ray diffraction and EXAFS. ${ }^{20,58}$ Units are in $\AA$, error are $\pm 0.05 \AA$ for theoretical values and $\pm 0.02 \AA$ for experimental the values

\begin{tabular}{lllll}
\hline & revPBE-D3 & RPA & SCAN & Experiment \\
\hline $\mathrm{Na}^{+}$ & 2.51 & - & 2.36 & 2.38 \\
$\mathrm{~K}^{+}$ & 2.98 & 2.86 & 2.78 & 2.76
\end{tabular}

have previously demonstrated that the PMF calculated with the BLYP-D2 functional leads to reasonably good agreement with experimental osmotic/activity coefficients. ${ }^{61}$

Different functionals may describe anion-water and cationwater interactions with significantly different accuracy. ${ }^{24}$ GGAs appear to already adequately reproduce the structure of halide ions such as iodide. ${ }^{62}$ But the accuracy of SCAN when applied to anions should be tested in future work.

One possibility is that there is a substantial cancellation of errors for example between cation-water and water-water interactions leading to the good agreement with hydration structure. We consider this to be unlikely as we demonstrate below using a new measure of the accuracy of the ion-water interactions that SCAN accurately reproduces ion-water interactions with the errors being below $k_{\mathrm{B}} T$. However, given the larger error for the pure water structure, this remains a possible issue with the SCAN functional.

Previous studies have determined that the solvation structure of the potassium ion is one of the most challenging cases to reproduce using GGA functionals. ${ }^{16}$ To explore this problem we have also simulated the potassium ion treating electron correlation at the level of the RPA employing PBE orbitals as input. ${ }^{40,41}$ Due to the significant computational costs of this functional we have not yet attempted the simulation of the sodium ion with this method. Fig. 2 suggests that the RPA functional performs substantially better than the GGA in comparison with the experimental peak position. The $\mathrm{K}-\mathrm{O}$ distance is somewhat larger than the experimental value, although this may be due to a limited trajectory size. Nevertheless, given that we used the converged GGA simulation to generate the initial conditions it is clear that the RPA is producing much better results more in line with EXAFS experiment. Interestingly the RPA is also more structured than both the GGA or the meta-GGA. Given the excellent agreement of RPA results for water under bulk homogeneous conditions, ${ }^{63}$ overall the performance of the RPA is satisfactory and hints that properly converged correlated wavefunction methods may well out-perform lower rungs of Jacob's Ladder but at a large computational overhead.

An additional key structural property that is relevant to our understanding of solvation thermodynamics is the hydration 

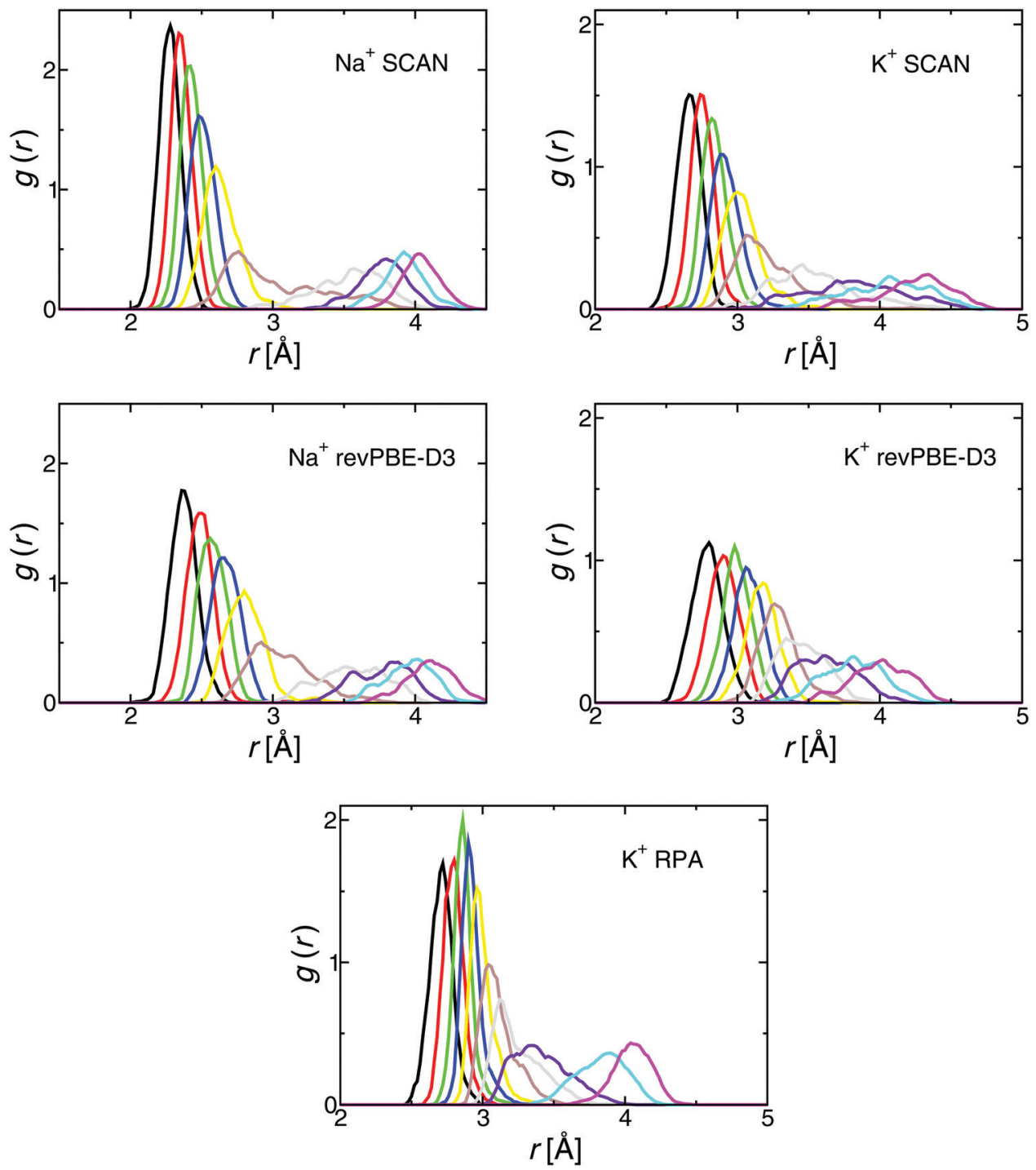

Fig. 3 Incremental RDFs of the sodium and potassium ions in water with the SCAN, revPBE-D3 and RPA functional. Black gives the distribution of the closest molecule, red the second closest etc.

number of the ion. However, this quantity is not well defined for ions having interstitial water molecules that lie between the first and second hydration shells. The best way to understand the ion hydration and its model dependencies is through the examination of the incremental RDFs that separate the pair distribution into contributions from the closest set of water molecules. Fig. 3 shows this quantity for the sodium and potassium ions using the three different functionals. It demonstrates that the seventh water molecule for sodium almost always occupies the second hydration shell. For potassium the sixth, seventh and eight water molecules bridge the first and second hydration shells blurring the distinction between hydration layers.

Fig. 4 provides an analysis of the average position and spread of individual water molecules about the ions using the SCAN functional. There are substantial similarities between the two ions. For instance, it is clear that both ions have five relatively tightly bound water molecules and a more diffuse sixth water molecule that occupies both the first and second hydration shells. ${ }^{20}$ In contrast, water molecules beyond the first shell show qualitatively different behaviour for the two ions. With the seventh being more localised for sodium whereas the sixth, seventh and eighth are all less localised for potassium.

The hydration number, which is defined as the integral of the RDF from zero to the first minima, gives values of $5.6 \pm 0.2$ for $\mathrm{Na}^{+}$and $7.5 \pm 0.8$ for $\mathrm{K}^{+}$with the SCAN functional. The large uncertainty in the potassium ion coordination number is due to the broad and flat minimum of the RDF and is the result of the fact that this ion has less well differentiated hydration layers. These results are consistent with XAFS and XRD data which gives a sodium hydration number of 5.4 to $5.9 .^{20}$

\section{EXAFS analysis}

From an implicit set of molecular interactions, an associated ensemble of configurations is generated from an MD trajectory. From this ensemble, the collective properties and responses of 


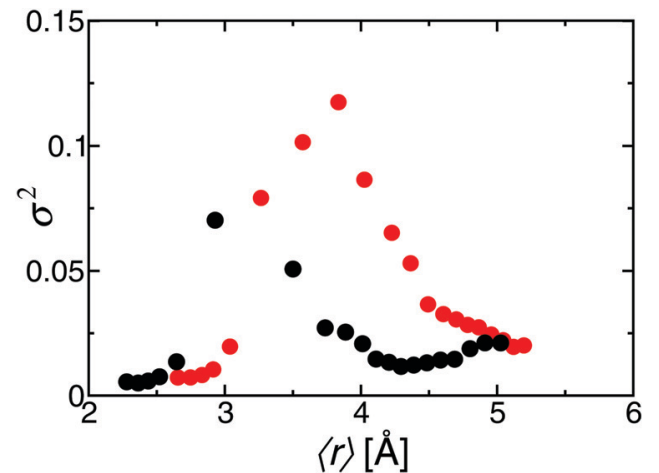

Fig. 4 Analysis of the incremental RDFs for sodium (black) and potassium (red) with the SCAN functional. Each point gives the average position and fluctuation in the position (the Debye-Waller factor) of one water molecule in the incremental RDF.

the system can be determined. In particular, it is possible to quantitatively generate the X-ray absorption fine structure spectra which is a unique spectral signature associated with the generated ensemble. (In this work we concentrate on the 'extended' region of the signal, referred to as EXAFS.) This provides a comprehensible benchmark of our description of molecular interactions, giving us confidence in its ability to generate accurate ensembles and predict phenomena that are not directly measurable.

EXAFS is an effective probe of the local solvent structure about a solute photo-electron source. The signal is most sensitive to the solute - nearest solvent distance and its fluctuations which are commonly defined by the Debye-Waller factor of the solutesolvent vibrations or $\sigma^{2}$. In Fig. 4, the waters at the first five

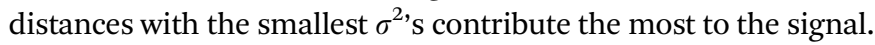
Although the trajectory ensemble also includes weaker EXAFS contributions for water beyond the fifth.

In order to connect to experimental measurements, we take configurations from a canonical ensemble, corresponding to the density and temperature of the measurement that is determined from the SCAN description of the molecular interaction. For each of the more than 3000 individual configurations, we generate an EXAFS signal using the feff9 code developed by Rehr and coworkers. ${ }^{64}$ We take the mean of this set of configurations to generate a signal corresponding to the ensemble. In Fig. 5 we compare the $k^{2}$ weighted fine structure, $k^{2} \chi(k)$, vs. $k$ (in $\AA^{-1}$ ), generated in this manner to the experimental measurement. ${ }^{20,58}$

For both $\mathrm{K}^{+}$and $\mathrm{Na}^{+}$we observe nearly quantitative agreement with the measured signal. For the $|\chi(r)|$ 's we recover both the main peak position at $2 \AA$ and the width. In addition, we recover the multiple scattering contributions between $3 \AA$ and $5 \AA$. The $k^{2} \chi(k)$ agreement in amplitude and frequency from 1.5 to $9 \AA^{-1}$ gives us confidence in the consistency between measurement and simulation.

For $\mathrm{Na}^{+}$the mean square variation in the distance obtained from the full width at half maximum of the Gaussian fit can be determined accurately from experimental XRD to be $0.020 \AA^{2}$. This agrees perfectly with the value determined from the simulation data of SCAN. This can be clearly seen in Fig. 4 .

\section{Discussion and error analysis}

SCAN has a more sophisticated description of exchange and correlation than revPBE-D3 and so in principle we should expect it to give more accurate structural agreement. This appears to be true for the cation's hydration structure but apparently this comes at the expense of the quality of the pure water oxygenoxygen RDF. It is important to understand the underlying reasons for this discrepancy. It is not uncommon for DFT functionals at similar levels of theory to predict substantially different structural details. ${ }^{29,38}$ This is also a challenge in the area of classical force field development where force field dependence remains a significant issue.

Obviously, the structures are determined by the energetics and so it should be possible to relate the structural performance of a given functional to how accurately it matches high-level correlated quantum mechanical calculations. However, there are two challenges to doing this. First, higher level methods cannot feasibly be applied to the larger sized systems examined here so energies of small clusters must be used as a proxy and it is not obvious which clusters are the best for comparison. Second, it is not clear which energy metric is best suited to capture the performance of a functional, as there are various choices of how to define the error in the energies. Identifying a reliable and inexpensive method that can predict the ability of a DFT functional to reproduce structural properties would be extremely useful in order to avoid wasting substantial amounts of computational time simulating large systems with functionals that will not work.

A standard method used in the development of forcefield parameters is to fit to ion-water binding energies calculated with a high-level of quantum mechanical theory. ${ }^{11,22,65,66}$ Similarly, it is common to use ion-water binding energies to benchmark and compare different levels of DFT theory. ${ }^{22,66}$

Often only very small clusters are considered such as dimers or trimers and the structures of these clusters are minimum energy structures or artificial perturbations about this minimum. In our view this is not the best choice, as the minimum energy structure of an ion-water dimer, for instance, will also be very different from the typical structures that water molecules occupy around ions under the conditions of bulk solvation, which can be quite far from an energy minima. Therefore, it is preferable to extract from the simulation a set of clusters representing the closest $n$ waters about the ion. Larger water clusters than dimers and trimers will be more relevant as they will incorporate the desired ion-water and water-water many-body effects. For these reasons we therefore evaluate clusters of water molecules surrounding a central ion or water molecule that have been extracted from the simulation.

The second issue is selection of the most appropriate energy value. The most commonly used value is simply the total binding energy of the cluster relative to its monomers separated in vacuum. For the case of a pure water cluster this is given by:

$$
E_{\text {mon }}=\left(\mathrm{H}_{2} \mathrm{O}\right)_{n+1}-(n+1) E_{\mathrm{H}_{2} \mathrm{O}}
$$

For the case of the cation cluster it is given by:

$$
E_{\mathrm{mon}}=E_{\mathrm{C}^{+}\left(\mathrm{H}_{2} \mathrm{O}\right)_{n}}-E_{\mathrm{C}^{+}}-n E_{\mathrm{H}_{2} \mathrm{O}}
$$



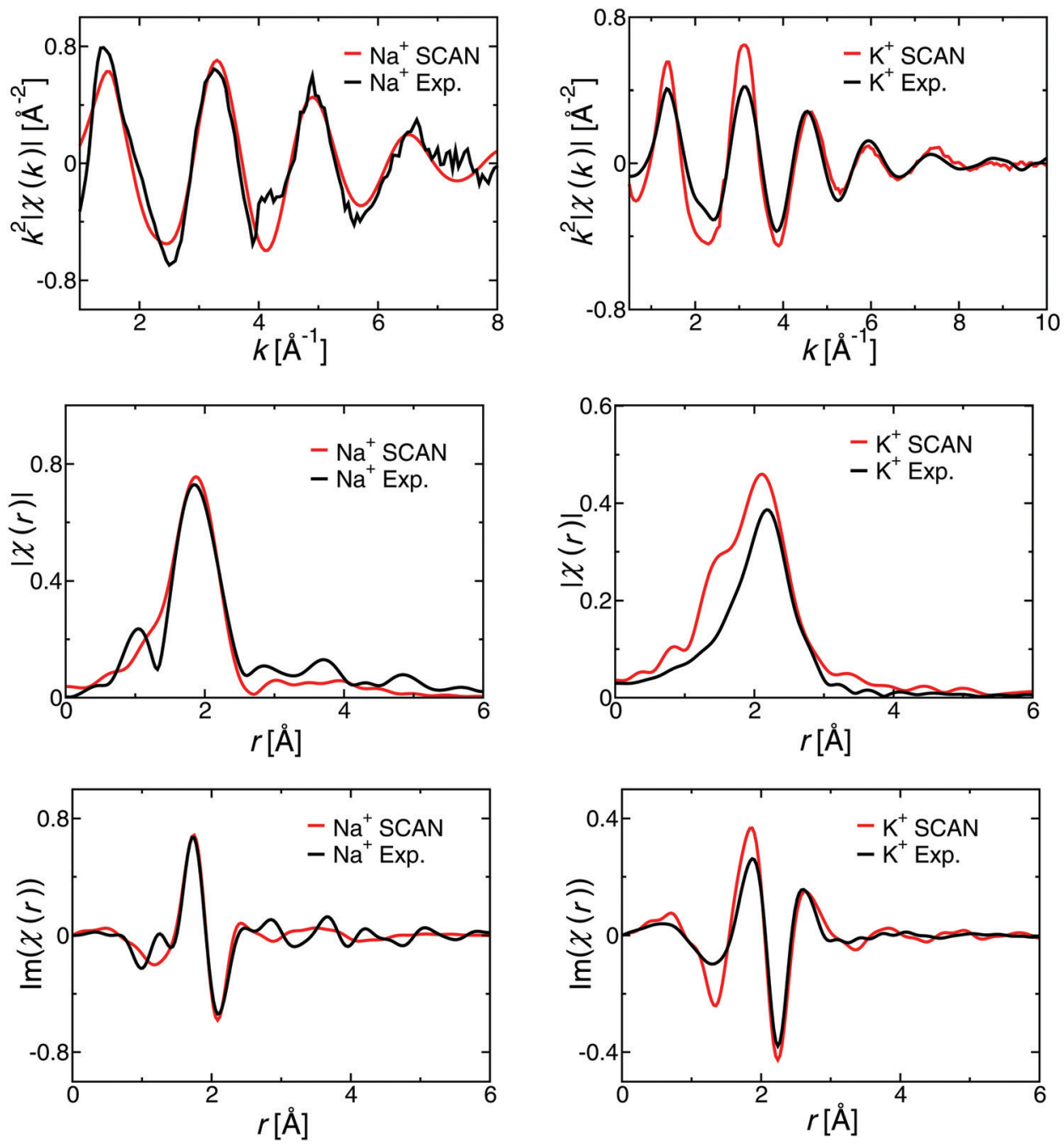

Fig. 5 EXAFS calculations compared with experiment for sodium (left) and potassium (right). Experimental data for potassium is from ref. 58. Experimental data for sodium is from ref. 20 after revision for self-absorption (see Experimental details).

The "mon" subscript indicates that the monomer energies are used for the separated molecules. This quantity will depend on the cluster size.

When comparing with multiple structures, it is customary to calculate the RMSD of this binding energy between the DFT functional and a higher level of theory such as MP2. This is given by:

$$
\mathrm{RMSD}_{\mathrm{mon}}=\sqrt{\frac{1}{N} \sum_{N}\left(E_{\mathrm{mon}}^{\mathrm{MP2}}-E_{\mathrm{mon}}^{\mathrm{DFT}}\right)^{2}}
$$

where the sum is over $N$ frames extracted from the simulation and $E_{\text {mon }}^{\mathrm{MP} 2}$ and $E_{\mathrm{mon}}^{\mathrm{DFT}}$ are $E_{\mathrm{mon}}$ calculated at the MP2 and DFT level of theory respectively. We use the MP2 level of theory with augmented correlation consistent quadruple-zeta basis sets.

Table 2 shows the results of calculating this $\mathrm{RMSD}_{\text {mon }}$ quantity. For the pure water cluster, revPBE-D3 outperforms SCAN significantly with an error of only $10.8 \mathrm{~kJ} \mathrm{~mol}^{-1}$ or about $1 \mathrm{~kJ} \mathrm{~mol}^{-1}$ per water molecule. This error for SCAN is substantially
Table 2 RMSD error in cluster binding energies $\left(E_{\text {mon }}\right)$ for DFT compared with MP2 (aug-cc-pVQZ). Units are in $\mathrm{kJ} \mathrm{mol}^{-1}$. All structures are extracted from simulation with the SCAN functional

\begin{tabular}{llcr}
\hline Cluster & DFT functional & RMSD $_{\text {mon }}$ & $\left\langle E_{\text {mon }}\right\rangle$ \\
\hline$\left(\mathrm{H}_{2} \mathrm{O}\right)_{9}$ & revPBE-D3 & 10.8 & -91.1 \\
$\left(\mathrm{H}_{2} \mathrm{O}\right)_{9}$ & SCAN & 27.6 & -109.3 \\
$\left(\mathrm{H}_{2} \mathrm{O}\right)_{9}$ & MP2 & 0 & -82.0 \\
$\mathrm{Na}^{+}\left(\mathrm{H}_{2} \mathrm{O}\right)_{8}$ & revPBE-D3 & 17.2 & -356.8 \\
$\mathrm{Na}^{+}\left(\mathrm{H}_{2} \mathrm{O}\right)_{8}$ & SCAN & 25.3 & -368.2 \\
$\mathrm{Na}^{+}\left(\mathrm{H}_{2} \mathrm{O}\right)_{8}$ & MP2 & 0 & -343.1 \\
$\mathrm{~K}^{+}\left(\mathrm{H}_{2} \mathrm{O}\right)_{8}$ & revPBE-D3 & 14.3 & -277.7 \\
$\mathrm{~K}^{+}\left(\mathrm{H}_{2} \mathrm{O}\right)_{8}$ & SCAN & 13.0 & -279.9 \\
$\mathrm{~K}^{+}\left(\mathrm{H}_{2} \mathrm{O}\right)_{8}$ & RPA & 13.6 & -254.0 \\
$\mathrm{~K}^{+}\left(\mathrm{H}_{2} \mathrm{O}\right)_{8}$ & MP2 & 0 & -267.2
\end{tabular}

larger at $27.6 \mathrm{~kJ} \mathrm{~mol}^{-1}$. This over-binding of the pure water clusters is consistent with previous work ${ }^{67}$ and is a potential explanation for the over-structuring of water observed with the SCAN functional (see Fig. 1).

Table 2 also shows that the RMSD mon totally fails to explain why SCAN is better for describing the hydration structure 
around the cation clusters. It predicts very similar errors for the potassium clusters for the three different choices and actually slightly larger errors for the sodium cluster with SCAN. The reason for this is likely that $\mathrm{RMSD}_{\text {mon }}$ depends mostly on the error in the water-water interactions but not on the cation-water interactions. We propose that the cation-water interactions are more important for correctly reproducing the hydration structure around an ion than the water-water interactions. This is because ion-water interactions are significantly stronger than water-water interactions due to the strong charge dipole interaction. An additional issue is that $\mathrm{RMSD}_{\text {mon }}$ will increase as the number of water molecules in the extracted cluster is increased.

A better metric for predicting how well water structure is reproduced specifically around the ion may be:

$$
E_{\text {clust }}=E_{\mathrm{C}^{+}\left(\mathrm{H}_{2} \mathrm{O}\right)_{n}}-E_{\mathrm{C}^{+}}-E_{\left(\mathrm{H}_{2} \mathrm{O}\right)_{n}} \text {. }
$$

Here the $E_{\left(\mathrm{H}_{2} \mathrm{O}\right)_{n}}$ is computed by simply removing the ion and recomputing the energy. This expression gives the binding energy of the ion to the water, with no contribution from the water-water interaction. It is denoted with the "clust" subscript to indicate that the water molecules are kept together in a cluster. This is also a very important quantity as it is the definition of the energy used in the potential distribution theorem to determine the single ion solvation free energy. ${ }^{32}$

The RMSD error in the cluster binding energy can be expressed in the same way as in the monomer case (eqn (3)). However, this is not the optimal choice for defining the error if the goal is to accurately reproduce the structural properties of the ion in water using DFT. It is possible to have large average errors in the total binding energy and still accurately reproduce the structural details. This is because the water structure is actually determined by forces (derivatives of the energy) rather than the pure energies and thus a large error in the total binding energy will not affect the structural properties directly if there is not a significant variation in the error of the binding energies from structure to structure.

There are several reasons why there could be large relatively fixed errors in the absolute binding energy which do not degrade the structural agreement significantly. One example, is basis set superposition error (BSSE). Standard basis sets used in condensed phase simulation are not at the complete basis set limit as running simulations at the BSSE limit is too computationally expensive. BSSE is known to be a substantial issue when calculating absolute binding free energies of clusters. However, this effect is much less significant when evaluating the relative difference in energy between similar clusters due to cancellation of error. Because it is these relative energy changes that determine the structural distributions, structural properties will be much less susceptible to BSSE. ${ }^{39}$

Therefore, assuming the ions are never isolated in vacuum, the average error in the total binding energy will not be the best indicator of a DFT functionals ability to reproduce structural details. An alternative way of seeing this is that the relative probability of two states (A and $\mathrm{B}$ ) is given by the ratio of the
Boltzmann factors which will not be altered by large constant error terms. That is:

$$
\frac{\mathrm{e}^{-\beta E_{\mathrm{A}}+E_{\text {error }}}}{\mathrm{e}^{-\beta E_{\mathrm{B}}+E_{\text {error }}}}=\frac{\mathrm{e}^{-\beta E_{\mathrm{A}}}}{\mathrm{e}^{-\beta E_{\mathrm{B}}}}
$$

We suggest that a more effective quantity to compare the quality of the DFT results is the standard deviation of the mean signed error. This is given by:

$$
\mathrm{SD}-\mathrm{ME}_{\text {clust }}=\sqrt{\frac{1}{N-1} \sum_{N}\left(\left(E_{\text {clust }}^{\mathrm{MP} 2}-E_{\text {clust }}^{\mathrm{DFT}}\right)-\left\langle E_{\text {clust }}^{\mathrm{MP} 2}-E_{\text {clust }}^{\mathrm{DFT}}\right\rangle\right)^{2}}
$$

The angle brackets term is the average error in the DFT binding energy compared with MP2 over the $N$ structures sampled. This expression captures the variation of the error from structure-tostructure. If this quantity is very small throughout the entire simulation, then the structural details should be perfectly reproduced regardless of whether the total binding energy is accurate or not.

It is true that many properties, such as single ion solvation free energies, are sensitive to the accuracy of the absolute binding free energy not just the variation in the error. However, once accurate structures are obtained, standard perturbative methods can be used to estimate absolute binding energies in situations where this quantity is of importance. See ref. 32 for an example of this.

Table 3 shows computed values for various estimates of the error. If we compute the standard deviation of the mean signed error using the $E_{\text {clust }}$ quantity given above (SD-ME $E_{\text {clust }}$ ). We can see that the error going from revPBE-D3 to SCAN is reduced by a factor of $3\left(6.7 \mathrm{~kJ} \mathrm{~mol}^{-1}\right.$ to $\left.2.0 \mathrm{~kJ} \mathrm{~mol}^{-1}\right)$ for $\mathrm{Na}\left(\mathrm{H}_{2} \mathrm{O}\right)_{8}$ clusters and by a factor of $5\left(7.9 \mathrm{~kJ} \mathrm{~mol}^{-1}\right.$ to $\left.1.6 \mathrm{~kJ} \mathrm{~mol}^{-1}\right)$ for $\mathrm{K}\left(\mathrm{H}_{2} \mathrm{O}\right)_{8}$ clusters.

The resulting variation with the SCAN is reduced below "chemical accuracy" (4.2 $\left.\mathrm{kJ} \mathrm{mol}^{-1}\right)$ and even thermal noise $\left(2.4 \mathrm{~kJ} \mathrm{~mol}^{-1}\right)$ levels. In contrast, it is larger than both of these thresholds for revPBE-D3 explaining the difference in performance in comparison with experimental structural properties. The $\mathrm{RMSD}_{\text {clust }}$ error for the $\mathrm{Na}^{+}\left(\mathrm{H}_{2} \mathrm{O}\right)_{8}(\mathrm{QZ})$ case actually goes up from revPBE-D3 to SCAN demonstrating that this is not a good estimate of the error if reproducing structural properties is the aim. It is clear that the error using the double zeta level of theory for the MP2 calculations shows a less dramatic shift which is not surprising considering there will be error associated with the smaller basis set. 32 water molecule clusters were also computed showing that $\mathrm{SD}-\mathrm{ME}_{\text {clust }}$ does not change significantly for larger cluster sizes. SD-ME $\mathrm{M}_{\text {clust }}$ is quite similar between RPA and SCAN. This suggests that the differences in hydration structure are likely due to convergence issues as conjectured earlier instead of an inherent problem with the RPA energy calculations.

Applying the SCAN functional to examine other cations such as $\mathrm{Cs}^{+}$and comparing with many body molecular models as carried out in ref. 68 are important future generalisations of this work. 
Table 3 Mean unsigned error in the cluster binding energies and fluctuations in the mean error. RMSD indicates the root mean squared deviation error estimate, whereas SD-ME indicates the standard deviation in the mean signed error. The DZ or QZ indicates whether double zeta or quadruple zeta basis sets were used for the MP2 level calculation. Units are in $\mathrm{kJ} \mathrm{mol}^{-1}$

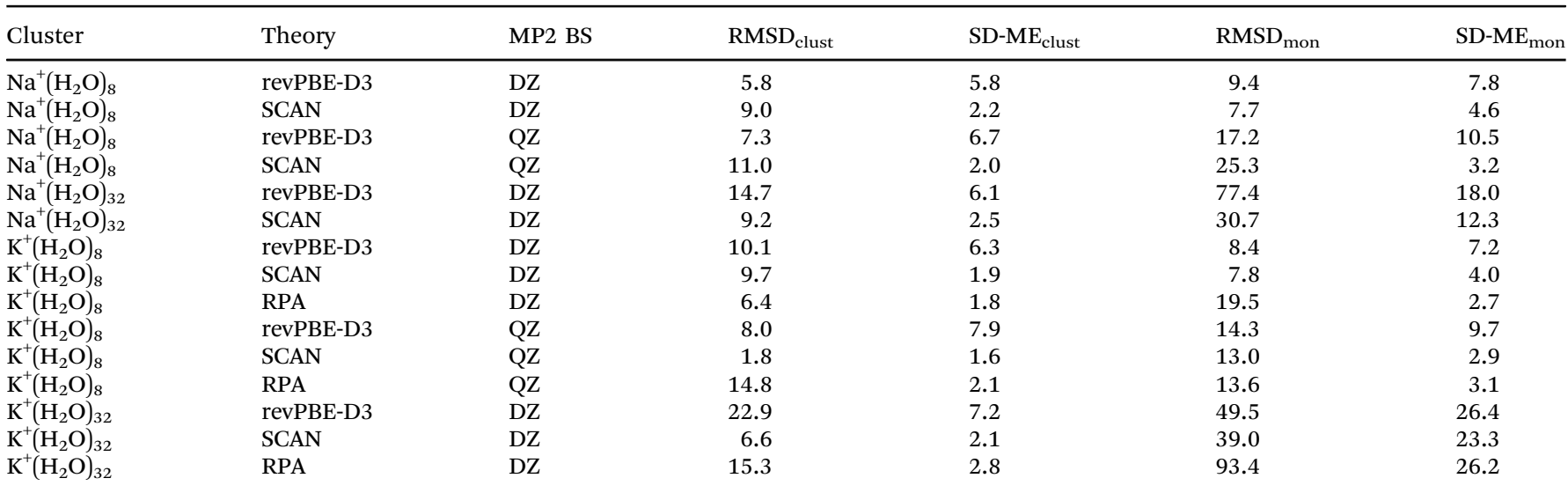

\section{Conclusion}

In summary, we have demonstrated that the newly developed SCAN functional can accurately reproduce fundamental structural properties of water around the sodium and potassium ions that standard GGA functionals fail to reproduce. However, the inability of SCAN to perfectly reproduce the structural properties of bulk water is highlighted. We present evidence that higher level wavefunction-based methods such as RPA provide the correct physics to reproduce experimental measurements considered herein. However, the hydration structure cannot be accurately reproduced using standard computational resources. We have argued that the fluctuation of the mean signed error of the ionwater cluster binding energy should be used to assess the quality of a DFT functional or forcefield and that this quantity explains the improved performance of the SCAN functional over revPBE-D3 in describing the hydration structure are $\mathrm{Na}^{+}$and $\mathrm{K}^{+}$.

\section{Computational details}

\section{$A b$ initio simulations}

For the revPBE and SCAN functionals, Born-Oppenheimer ab initio molecular dynamics simulations within the $N V T$ (at $300 \mathrm{~K}$ ) ensemble using periodic boundary conditions are performed within the CP2K simulation suite (http:www.cp2k.org) containing the QuickStep module for the DFT calculations. ${ }^{39}$ The D3 dispersion correction due to Grimme ${ }^{28}$ was used for revPBE. A 0.5 fs time step was used. We used a double $\zeta$ basis set that has been optimized for the condensed phase ${ }^{69}$ in conjunction with GTH pseudopotentials ${ }^{44}$ using a 400 Ry cutoff for the auxiliary plane wave basis for the revPBE-D3 simulations and a 1200 Ry cutoff for the SCAN simulations. ${ }^{38,70}$ The pseudopotentials for the oxygen atom were reoptimized for the SCAN functional using the ATOM code of CP2K. A NoséHoover thermostat was attached to every degree of freedom to ensure equilibration. ${ }^{71}$ The energies were accumulated for $\approx 12 \mathrm{ps}$ after $3 \mathrm{ps}$ of equilibration. The sodium and potassium simulations for revPBE-D3 and SCAN consisted of one ion in a box of 96 water molecules of dimensions $14.3^{3} \AA^{3}$ corresponding to a water density of $1 \mathrm{~g} \mathrm{~cm}^{-3}$.

For the RPA simulations of potassium the procedure outlined by Del Ben et al. ${ }^{40,41}$ was used. The simulation box consisted of 63 water molecules and a single potassium ion with dimensions of $12.42^{3} \AA^{3}$. Basis sets of correlation consistent triple zeta quality were used including new basis sets for the potassium. The cutoff is set to $800 \mathrm{Ry}$. Core electrons are replaced by pseudopotentials that have been parametrized for the PBE functional. The Kohn-Sham PBE orbitals were used as input for the RPA calculation. The Monte Carlo (MC) simulations have been performed employing the same setup as given in ref. 63, with $T=295 \mathrm{~K}$ and $p=1$ bar. The MC efficiency is improved with the presampling of moves, the approximated potential is calculated using the revPBE-D3 functional. The initial configuration has been equilibrated with a $15 \mathrm{ps} N V T$-MD run at the experimental density using the revPBE-D3 level. The statistics from the MC simulations were accumulated for 6100 frames after equilibration for 500 .

$\mathrm{ORCA}^{72}$ was used to calculate the cluster energies at the MP2 level of theory. Clusters of 8 and 32 water molecules were used in the cluster correction calculation with 50 frames extracted from the 15 ps trajectory with the SCAN functional. The aug-cc-p $x \mathrm{Z}$ basis set was used for the oxygen and hydrogen atoms. ${ }^{73}$ Similarly, the cc-pCVxZ basis set was used for the sodium ion ${ }^{74}$ and the cc-pwC $x \mathrm{Z}$ basis set for the potassium ion. ${ }^{75} x$ was either $\mathrm{D}$ or Q. Frozen cores were used for the MP2 calculations. RI-MP2 was used for the 32 water cluster calculations using automatically generated auxiliary basis functions. For the revPBE-D3 and SCAN cluster energy calculations CP2K was used with the periodicity none option and a larger cell size to remove any box size dependence. Otherwise, the same parameters, basis sets etc. as the simulation were used.

\section{Experimental details}

\section{EXAFS}

The sodium K-edge (1070.8 eV) EXAFS experiments were carried out at the Phoenix II, elliptical undulator beamline at the Swiss 
Light Source (SLS) at the Paul Scherrer Institute (PSI), Switzerland ${ }^{20}$ while the potassium K-edge EXAFS measurements were taken at the Sector 20 bending-magnet beamline of the Advanced Photon Source at Argonne National Laboratory. ${ }^{58}$

The measurements for $\mathrm{K}^{+}$include a correction of the multielectron features. (see ref. 58). In addition, a single E0 shift is universally applied to the ensemble in order to account for small errors in the absolute edge energy $( \pm 0.2 \%$, or $2 \mathrm{eV})$ calculated by FEFF. This is accomplished by adjusting E0 to assure the convergence of the oscillations (as $k \rightarrow 0 \AA^{-1}$ ) between the predicted and the experimental spectra (for example, the peak at about $1.5 \AA^{-1}$ in Fig. 5). In Fig. 5 we Fourier transform the $\chi(k)$ to construct $\chi(r)$. The transform range and settings are applied consistently to both the measurement and the simulation. The EXAFS $\chi(r)$ consists of a distribution of scattering distances in which the true distances have been shifted by the interaction of the scattered photoelectron with the underlying charge density on the nearby atom. This interaction is accounted for exactly in the multiple scattering code, FEFF. Experimental measurement of these systems is often complicated due to issues associated with multi electronelectron excitations. ${ }^{58}$

In the current work, we carve out clusters of the nearest 10 water molecules to the cation to use as input for EXAFS calculation using default settings of the FEFF9 code. For the $\mathrm{K}^{+}$ and $\mathrm{Na}^{+}$systems, every 10 th configuration was chosen to make up the ensemble, this gives 3055 configurations for $\mathrm{K}^{+}$and 3029 configurations for $\mathrm{Na}^{+}$. The ensemble averaged $\chi(k)$ signal is recovered from this average of configurations. Next, we take the Fourier transform of the $k^{2} \chi(k)$ signal to generate the $|\chi(R)|$ and $\operatorname{Im}[\chi(R)]$ signals. The $\mathrm{Na}^{+}$EXAFS spectrum in Fig. 5 was a modified version from an uncorrected spectrum previously reported. ${ }^{20}$ The absorption edge for $\mathrm{Na}(1071 \mathrm{eV})$ is quite low and achieving a linear response of the EXAFS detectors and the sample over the entire energy span $(+300 \mathrm{eV})$ is quite challenging. The factors affecting the energy-dependent response were extensively evaluated including responses of the incident beam detector, I0, the fluorescence detector, IF, and the attenuation length of the sample. These factors were approximately corrected by applying an energy-dependent normalization using the pre- and post-edge normalization lines rather than division by a constant edge-step value at the absorption edge. This methodology has been previously described. ${ }^{76}$

When compared to DFT simulations of simple anions and doubly charged cations, where the accuracy is quite high, it is has been a challenge to identify the correct combination of molecular interaction and statistical mechanical sampling to reproduce the measured EXAFS signals for the monovalent $\mathrm{Na}^{+}$ and $\mathrm{K}^{+}$cations in aqueous solvation. It is a challenge to find a balance between the (i) strong cation-water interaction, (ii) the solvent-solvent interaction in the presence of the cation that disrupts the hydrogen-bonding network and (iii) the longrange interactions defining the bulk structure and response of water. The use of a single molecular framework to consistently describe each of these molecular responses remains a challenge.

\section{Conflicts of interest}

There are no conflicts to declare.

\section{Acknowledgements}

TTD, MG, GKS and CJM were supported by the U.S. Department of Energy, Office of Science, Office of Basic Energy Sciences, Division of Chemical Sciences, Geosciences, and Biosciences. MD Baer was supported by $\mathrm{MS}^{3}$ (Materials Synthesis and Simulation Across Scales) Initiative, a Laboratory Directed Research and Development Program PNNL. PNNL is a multiprogram national laboratory operated by Battelle for the U.S. Department of Energy. The RPA and SCAN calculation used resources of the National Energy Research Scientific Computing Center (NERSC), a U.S. Department of Energy Office of Science User Facility operated under Contract No. DE-AC02-05CH11231. Calculations were also performed on PNNL's Institutional Computing resource. The Na XAFS measurements were performed at the PHOENIX beamline of the Swiss Light Source, Paul Scherrer Institute, Villigen, Switzerland. JW and $\mathrm{JH}$ are supported by The National Centre of Competence in Research (NCCR) Materials Revolution: Computational Design and Discovery of Novel Materials (MARVEL) of the Swiss National Science Foundation (SNSF). XSZ and TTD acknowledge the Australian Research Council (ARC) funding via project number FL170100101. MDB is supported by the Center for Computational Study of Excited-State Phenomena in Energy Materials (C2SEPEM) and by the SciDAC Program on Excited State Phenomena in Energy Materials at the Lawrence Berkeley National Laboratory, which is funded by the U.S. Department of Energy, Office of Science, Basic Energy Sciences, Materials Sciences and Engineering Division under Contract No. DE-AC02-05CH11231, as part of the Computational Materials Sciences Program. This research used resources of the Advanced Photon Source, an Office of Science User Facility operated for the U.S. Department of Energy (DOE) Office of Science by Argonne National Laboratory and was supported under DOE under Contract No. DE-AC02-06CH11357, and the Canadian Light Source and its funding partners. This research was undertaken with the assistance of resources from QCIF (http://www.qcif.edu.au).

\section{References}

1 K. Kubota and S. Komaba, Review-practical issues and future perspective for Na-ion batteries, J. Electrochem. Soc., 2015, 162, A2538-A2550.

2 C. D. Wessells, S. V. Peddada, R. A. Huggins and Y. Cui, Nickel hexacyanoferrate nanoparticle electrodes for aqueous sodium and potassium ion batteries, Nano Lett., 2011, 11, 5421-5425.

3 Y. Liu, F. Fan, J. Wang, Y. Liu, H. Chen, K. L. Jungjohann, Y. Xu, Y. Zhu, D. Bigio and T. Zhu, et al., In situ transmission electron microscopy study of electrochemical sodiation and potassiation of carbon nanofibers, Nano Lett., 2014, 14, 3445-3452. 
4 Z. Jian, Z. Xing, C. Bommier, Z. Li and X. Ji, Hard carbon microspheres: potassium-ion anode versus sodium-ion anode, Adv. Energy Mater., 2016, 6, 1501874.

5 G. Åvall, J. Mindemark, D. Brandell and P. Johansson, Sodium-ion battery electrolytes: modeling and simulations, Adv. Energy Mater., 2018, 8, 1703036.

6 M. J. V. Clausen and H. Poulsen, Metallomics and the Cell, Metal Ions in Life Sciences, Springer, Dordrecht, 2013, vol. 12, pp. 41-67.

7 J. Peng, D. Cao, Z. He, J. Guo, P. Hapala, R. Ma, B. Cheng, J. Chen, W. J. Xie and X.-Z. Li, et al., The effect of hydration number on the interfacial transport of sodium ions, Nature, 2018, 557, 701-705.

8 L. X. Dang, J. E. Rice, J. Caldwell and P. A. Kollman, Ion solvation in polarizable water: molecular dynamics simulations, J. Am. Chem. Soc., 1991, 113, 2481-2486.

9 T.-M. Chang and L. X. Dang, Detailed study of potassium solvation using molecular dynamics techniques, J. Phys. Chem. B, 1999, 103, 4714-4720.

10 J. A. White, E. Schwegler, G. Galli and F. Gygi, The solvation of $\mathrm{Na}^{+}$in water: first-principles simulations, J. Chem. Phys., 2000, 113, 4668.

11 M. Carrillo-Tripp, H. Saint-Martin and I. Ortega-Blake, A comparative study of the hydration of $\mathrm{Na}^{+}$and $\mathrm{K}^{+}$with refined polarizable model potentials, J. Chem. Phys., 2003, 118, 7062-7073.

12 S. Varma and S. B. Rempe, Coordination numbers of alkali metal ions in aqueous solutions, Biophys. Chem., 2006, 124, 192-199.

13 S. S. Azam, T. S. Hofer, B. R. Randolf and B. M. Rode, Hydration of sodium(I) and potassium(I) revisited: a comparative QM/MM and QMCF MD simulation study of weakly hydrated ions, J. Phys. Chem., 2009, 113, 1827-1834.

14 C. $\mathrm{N}$. Rowley, The solvation structure of $\mathrm{Na}^{+}$and $\mathrm{K}^{+}$in liquid water determined from high level ab initio molecular dynamics simulations, J. Chem. Theory Comput., 2012, 8, 3526-3535.

15 A. Bankura, V. Carnevale and M. L. Klein, Hydration structure of salt solutions from ab initio molecular dynamics, J. Chem. Phys., 2013, 138, 014501.

16 A. Bankura, V. Carnevale and M. L. Klein, Hydration structure of $\mathrm{Na}^{+}$and $\mathrm{K}^{+}$from ab initio molecular dynamics based on modern density functional theory, Mol. Phys., 2014, 112, 1448-1456.

17 A. P. Gaiduk, C. Zhang, F. Gygi and G. Galli, Structural and electronic properties of aqueous $\mathrm{NaCl}$ solutions from $\mathrm{ab}$ initio molecular dynamics simulations with hybrid density functionals, Chem. Phys. Lett., 2014, 604, 89-96.

18 M. Soniat, D. M. Rogers and S. B. Rempe, Dispersion- and exchange-corrected density functional theory for sodium ion hydration, J. Chem. Theory Comput., 2015, 11, 2958-2967.

19 T. Ikeda and M. Boero, Role of van der Waals corrections in first principles simulations of alkali metal ions in aqueous solutions, J. Chem. Phys., 2015, 143, 194510.

20 M. Galib, M. D. Baer, L. B. Skinner, C. J. Mundy, T. Huthwelker, G. K. Schenter, C. J. Benmore, N. Govind and J. L. Fulton, Revisiting the hydration structure of aqueous Na, J. Chem. Phys., 2017, 146, 084504.
21 T. S. Hofer and P. H. Hünenberger, Absolute proton hydration free energy, surface potential of water, and redox potential of the hydrogen electrode from first principles: $\mathrm{QM} / \mathrm{MM} \mathrm{MD}$ freeenergy simulations of sodium and potassium hydration, J. Chem. Phys., 2018, 148, 222814.

22 M. Riera, A. W. Götz and F. Paesani, The i-TTM model for ab initio-based ion-water interaction potentials. II. Alkali metal ion-water potential energy functions, Phys. Chem. Chem. Phys., 2016, 18, 30334-30343.

23 M. Riera, N. Mardirossian, P. Bajaj, A. W. Götz and F. Paesani, Toward chemical accuracy in the description of ion-water interactions through many-body representations. Alkali-water dimer potential energy surfaces, J. Chem. Phys., 2017, 147, 161715.

24 B. B. Bizzarro, C. K. Egan and F. Paesani, On the nature of halide-water interactions: insights from many-body representations and density functional theory, J. Chem. Theory Comput., 2019, 15, 2983-2995.

25 T. A. Pham, Ab initio simulations of liquid electrolytes for energy conversion and storage, Int. J. Quantum Chem., 2018, 119, e25795.

26 J. P. Perdew, K. Burke and M. Ernzerhof, Generalized gradient approximation made simple, Phys. Rev. Lett., 1996, 77, 3865-3868.

27 Y. Zhang and W. Yang, Comment on "Generalized gradient approximation made simple”, Phys. Rev. Lett., 1998, 80, 890.

28 S. Grimme, J. Antony, S. Ehrlich and H. Krieg, A consistent and accurate $\mathrm{ab}$ initio parametrization of density functional dispersion correction (DFT-D) for the 94 elements $\mathrm{H}-\mathrm{Pu}$, J. Chem. Phys., 2010, 132, 154104.

29 M. Galib, T. T. Duignan, Y. Misteli, M. D. Baer, G. K. Schenter, J. Hutter and C. J. Mundy, Mass density fluctuations in quantum and classical descriptions of liquid water, J. Chem. Phys., 2017, 146, 244501.

30 M. Galib, G. K. Schenter, C. J. Mundy, N. Govind and J. L. Fulton, Unraveling the spectral signatures of solvent ordering in K-edge XANES of aqueous $\mathrm{Na}^{+}, J$. Chem. Phys, 2018, 149, 124503.

31 T. T. Duignan, M. D. Baer, G. K. Schenter and C. J. Mundy, Electrostatic solvation free energies of charged hard spheres using molecular dynamics with density functional theory interactions, J. Chem. Phys., 2017, 147, 161716.

32 T. T. Duignan, M. D. Baer, G. K. Schenter and C. J. Mundy, Real single ion solvation free energies with quantum mechanical simulation, Chem. Sci., 2017, 8, 6131-6140.

33 R. C. Remsing and A. J. Patel, Water density fluctuations relevant to hydrophobic hydration are unaltered by attractions, J. Chem. Phys., 2015, 142, 024502.

34 R. C. Remsing and J. D. Weeks, Role of local response in ion solvation: Born theory and beyond, J. Phys. Chem. B, 2016, 120, 6238-6249.

35 J. P. Perdew, A. Ruzsinszky, J. Tao, V. N. Staroverov, G. E. Scuseria and G. I. Csonka, Prescription for the design and selection of density functional approximations: More constraint satisfaction with fewer fits, J. Chem. Phys., 2005, 123, 062201. 
36 J. Sun, A. Ruzsinszky and J. Perdew, Strongly constrained and appropriately normed semilocal density functional, Phys. Rev. Lett., 2015, 115, 036402.

37 J. Sun, R. C. Remsing, Y. Zhang, Z. Sun, A. Ruzsinszky, H. Peng, Z. Yang, A. Paul, U. Waghmare and X. Wu, et al., Accurate first-principles structures and energies of diversely bonded systems from an efficient density functional, Nat. Chem., 2016, 8, 831-836.

38 Y. Yao and Y. Kanai, Free energy profile of $\mathrm{NaCl}$ in water: First-principles molecular dynamics with SCAN and $\omega \mathrm{B} 97 \mathrm{X}-\mathrm{V}$ exchange-correlation functionals, J. Chem. Theory Comput., 2018, 14, 884-893.

39 J. VandeVondele, M. Krack, F. Mohamed, M. Parrinello, T. Chassaing and J. Hutter, Quickstep: Fast and accurate density functional calculations using a mixed Gaussian and plane waves approach, Comput. Phys. Commun., 2005, 167, 103-128.

40 M. Del Ben, J. Hutter and J. VandeVondele, Probing the structural and dynamical properties of liquid water with models including non-local electron correlation, J. Chem. Phys., 2015, 143, 054506.

41 M. Del Ben, O. Schütt, T. Wentz, P. Messmer, J. Hutter and J. VandeVondele, Enabling simulation at the fifth rung of DFT: large scale RPA calculations with excellent time to solution, Comput. Phys. Commun., 2015, 187, 120-129.

42 M. Chen, H.-y. Ko, R. C. Remsing, M. F. Calegari, B. Santra and Z. Sun, Ab initio theory and modeling of water, Proc. Natl. Acad. Sci. U. S. A., 2017, 24-26.

43 L. Zheng, M. Chen, Z. Sun, H.-Y. Ko, B. Santra, P. Dhuvad and $\mathrm{X}$. Wu, Structural, electronic, and dynamical properties of liquid water by ab initio molecular dynamics based on SCAN functional within the canonical ensemble, J. Chem. Phys., 2018, 148, 164505.

44 S. Goedecker, M. Teter and J. Hutter, Separable dual-space Gaussian pseudopotentials, Phys. Rev. B: Condens. Matter Mater. Phys., 1996, 54, 1703-1710.

45 L. B. Skinner, C. Huang, D. Schlesinger, L. G. M. Pettersson, A. Nilsson and C. J. Benmore, Benchmark oxygen-oxygen pair-distribution function of ambient water from X-ray diffraction measurements with a wide Q-range, J. Chem. Phys., 2013, 138, 074506.

46 O. Marsalek and T. E. Markland, Quantum dynamics and spectroscopy of ab initio liquid water: the interplay of nuclear and electronic quantum effects, J. Phys. Chem. Lett., 2017, 8, 1545-1551.

47 M. D. Baer, C. J. Mundy, M. J. McGrath, I.-F. W. Kuo, J. I. Siepmann and D. J. Tobias, Re-examining the properties of the aqueous vapor-liquid interface using dispersion corrected density functional theory, J. Chem. Phys., 2011, 135, 124712.

48 A. D. Becke, Density-functional exchange-energy approximation with correct asymptotic behavior, Phys. Rev. A: At., Mol., Opt. Phys., 1988, 38, 3098-3100.

49 C. Lee, W. Yang and R. G. Parr, Development of the ColleSalvetti correlation-energy formula into a functional of the electron density, Phys. Rev. B: Condens. Matter Mater. Phys., 1988, 37, 785-789.
50 S. Grimme, Accurate description of van der Waals complexes by density functional theory including empirical corrections, J. Comput. Chem., 2004, 25, 1463-1473.

51 G. R. Medders, V. Babin and F. Paesani, Development of a "first-principles" water potential with flexible monomers. III. Liquid phase properties, J. Chem. Theory Comput., 2014, 10, 2906-2910.

52 M. Ceriotti, W. Fang, P. G. Kusalik, R. H. McKenzie, A. Michaelides, M. A. Morales and T. E. Markland, Nuclear quantum effects in water and aqueous systems: experiment, theory, and current challenges, Chem. Rev., 2016, 116, 7529-7550.

53 L. R. Pestana, O. Marsalek, T. E. Markland and T. HeadGordon, The quest for accurate liquid water properties from first principles, J. Phys. Chem. Lett., 2018, 9, 5009-5016.

54 J. Wiktor, F. Ambrosio and A. Pasquarello, Note: Assessment of the SCAN + rVV10 functional for the structure of liquid water, J. Chem. Phys., 2017, 147, 10-12.

55 Y. Yao, Advancing molecular dynamics simulations of aqueous ionic solutions, PhD thesis, University of North Carolina, 2018.

56 J. Hutter, M. Iannuzzi, F. Schiffmann and J. VandeVondele, CP2K: atomistic simulations of condensed matter systems, Wiley Interdiscip. Rev.: Comput. Mol. Sci., 2014, 4, 15-25.

57 R. Car and M. Parrinello, Unified approach for molecular dynamics and density-functional theory, Phys. Rev. Lett., 1985, 55, 2471-2474.

58 V. A. Glezakou, Y. Chen, J. L. Fulton, G. K. Schenter and L. X. Dang, Electronic structure, statistical mechanical simulations, and EXAFS spectroscopy of aqueous potassium, Theor. Chem. Acc., 2006, 115, 86-99.

59 H. Ohtaki and T. Radnai, Structure and dynamics of hydrated ions, Chem. Rev., 1993, 93, 1157-1204.

60 Y. Marcus, Effect of ions on the structure of water: structure making and breaking, Chem. Rev., 2009, 109, 1346-1370.

61 T. T. Duignan, M. D. Baer and C. J. Mundy, Ions interacting in solution: moving from intrinsic to collective properties, Curr. Opin. Colloid Interface Sci., 2016, 23, 58-65.

62 J. L. Fulton, G. K. Schenter, M. D. Baer, C. J. Mundy, L. X. Dang and M. Balasubramanian, Probing the hydration structure of polarizable halides: A multiedge XAFS and molecular dynamics study of the iodide anion, J. Phys. Chem. $B, 2010,114,12926-12937$.

63 M. Del Ben, M. Schönherr, J. Hutter and J. VandeVondele, Bulk liquid water at ambient temperature and pressure from MP2 theory, J. Phys. Chem. Lett., 2013, 4, 3753-3759.

64 J. J. Rehr, J. J. Kas, F. D. Vila, M. P. Prange and K. Jorissen, Parameter-free calculations of X-ray spectra with FEFF9, Phys. Chem. Chem. Phys., 2010, 12, 5503-5513.

65 G. Lamoureux and B. Roux, Absolute hydration free energy scale for alkali and halide ions established from simulations with a polarizable force field, J. Phys. Chem. B, 2006, 110, 3308-3322.

66 D. J. Arismendi-Arrieta, M. Riera, P. Bajaj, R. Prosmiti and F. Paesani, The i-TTM model for ab initio-based ion-water interaction potentials. 1 . halide-water potential energy functions, J. Phys. Chem. B, 2016, 120, 1822-1832. 
67 F. Paesani, Water: many-body potential from first principles (from the gas to the liquid phase), Handb. Mater. Model., 2018, 1-25.

68 D. Zhuang, M. Riera, G. K. Schenter, J. L. Fulton and F. Paesani, Many-body effects determine the local hydration structure of Cs + in solution, J. Phys. Chem. Lett., 2019, 10, 406-412.

$69 \mathrm{~J}$. VandeVondele and J. Hutter, Gaussian basis sets for accurate calculations on molecular systems in gas and condensed phases, J. Chem. Phys., 2007, 127, 114105.

70 G. Miceli, J. Hutter and A. Pasquarello, Liquid water through density-functional molecular dynamics: plane-wave vs atomicorbital basis sets, J. Chem. Theory Comput., 2016, 12, 3456-3462.
71 G. J. Martyna, M. L. Klein and M. Tuckerman, Nosé-Hoover chains: the canonical ensemble via continuous dynamics, J. Chem. Phys., 1992, 97, 2635-2643.

72 F. Neese, The ORCA program system, Wiley Interdiscip. Rev.: Comput. Mol. Sci., 2012, 2, 73-78.

73 T. H. Dunning, Jr., Gaussian basis sets for use in correlated molecular calculations. I. The atoms boron through neon and hydrogen, J. Chem. Phys., 1989, 90, 1007-1023.

74 D. E. Woon and T. H. Dunning, Jr., Gaussian basis sets for use in correlated molecular calculations. IV. Calculation of static electrical response properties, J. Chem. Phys., 1994, 100, 2975-2988.

75 K. Peterson, 2017, Private communication.

76 B. Ravel, 2016, https://bruceravel.github.io/demeter/docu ments/Athena/bkg/ednorm.html. 